\title{
Endoscopic sclerotherapy under balloon-assisted enteroscopy for hemorrhagic jejunal varices after choledocho-jejunostomy
}

Hemorrhage from varices in the jejunal loop after reconstruction of the biliary tract is quite rare. Portal hypertension due to extrahepatic portal vein stenosis or obstruction at the site of a previously performed choledocho-jejunostomy results in the formation of hepatoportal collaterals through the anastomosis, thus creating varices in the afferent loop that can rupture and hemorrhage [1,2]. We present endoscopic sclerotherapy under balloon-assisted enteroscopy for hemorrhagic jejunal varices after choledocho-jejunostomy ( $\vee$ Video 1 ).

A 67-year-old man was admitted to our hospital with recurrent massive gastrointestinal bleeding (GIB). He had undergone choledocho-jejunostomy (Rouxen-Y) for pancreaticobiliary maljunction 14 years earlier, and 12 years later, he had the first episode of GIB. At another hospital, it was revealed that the cause of hemorrhage was jejunal varices at the site of the choledocho-jejunostomy anastomosis, which appeared to have developed due to an extrahepatic portal vein obstruction.
A transileocolic vein obliteration utilizing a catheter and coils was carried out and resulted in successful hemostasis. On the arrival at our hospital, recurrent jejunal varices were revealed by enhanced computed tomography ( $\mathbf{F i g} \mathbf{1}$ ).

Balloon-assisted enteroscopy using a single-balloon endoscope (SIF-Q260; Olympus, Tokyo, Japan) was carried out. It showed the coils, which were exposed ( $\mathbf{F i g . 2 a ) ~ n e a r ~ t o ~ t h e ~ s i t e ~ o f ~ t h e ~}$ choledocho-jejunostomy anastomosis ( Fig.2b). Jejunal varices with a fibrin plug were also revealed (\$ Fig. $\mathbf{2 c}$ ). We performed endoscopic injection sclerotherapy (EIS) using a mixture of $1.5 \mathrm{~mL}$ $\mathrm{N}$-butyl-2-cyanoacrylate and $0.5 \mathrm{~mL} \mathrm{li-}$ piodol at the jejunal varices ( $\mathbf{F i g} \mathbf{2 d}$ ). After EIS, the injectate could be seen occupying the jejunal varices ( $>$ Fig.3). There were no adverse events concerning the EIS treatment. From then on, no GIB episodes were encountered.

We conclude that EIS using balloonassisted enteroscopy can be an effective, easy, and safe treatment selection for hemorrhagic jejunal varices.

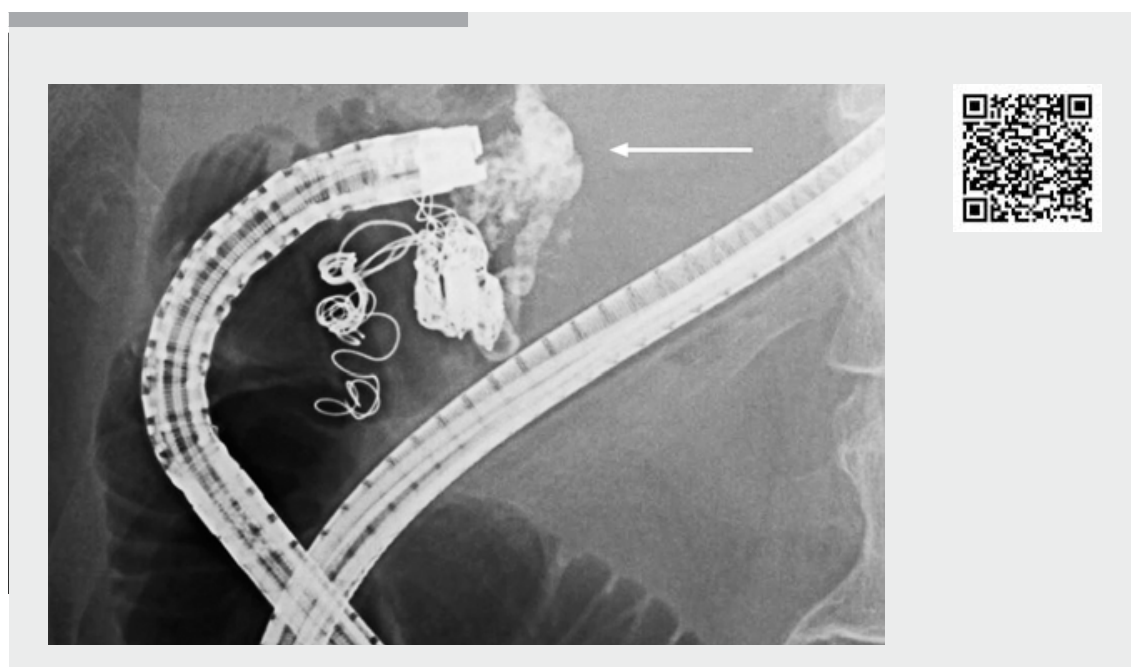

Video 1 Endoscopic sclerotherapy under balloon-assisted enteroscopy for hemorrhagic jejunal varices after choledocho-jejunostomy.
Endoscopy_UCTN_Code_TTT_1AP_2AD

Competing interests

None

The authors

Kota Takashima, Shigenaga Matsui, Yoriaki Komeda, Tomoyuki Nagai, Sakurai Toshiharu, Hiroshi Kashida, Masatoshi Kudo

Department of Gastroenterology and Hepatology, Kindai University Faculty of Medicine, Osaka, Japan

\section{Corresponding author}

Shigenaga Matsui, MD, PhD

Department of Gastroenterology and Hepatology, Kindai University Faculty of Medicine, 377-2 Ohno-Higashi, Osaka 589-8511, Japan

Fax: +81-723-672880

ma2i@med.kindai.ac.jp

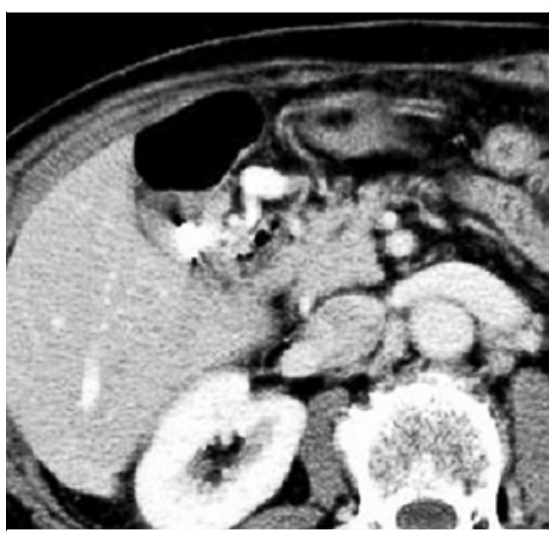

- Fig. 1 Enhanced computed tomography showing jejunal varices (arrow). 

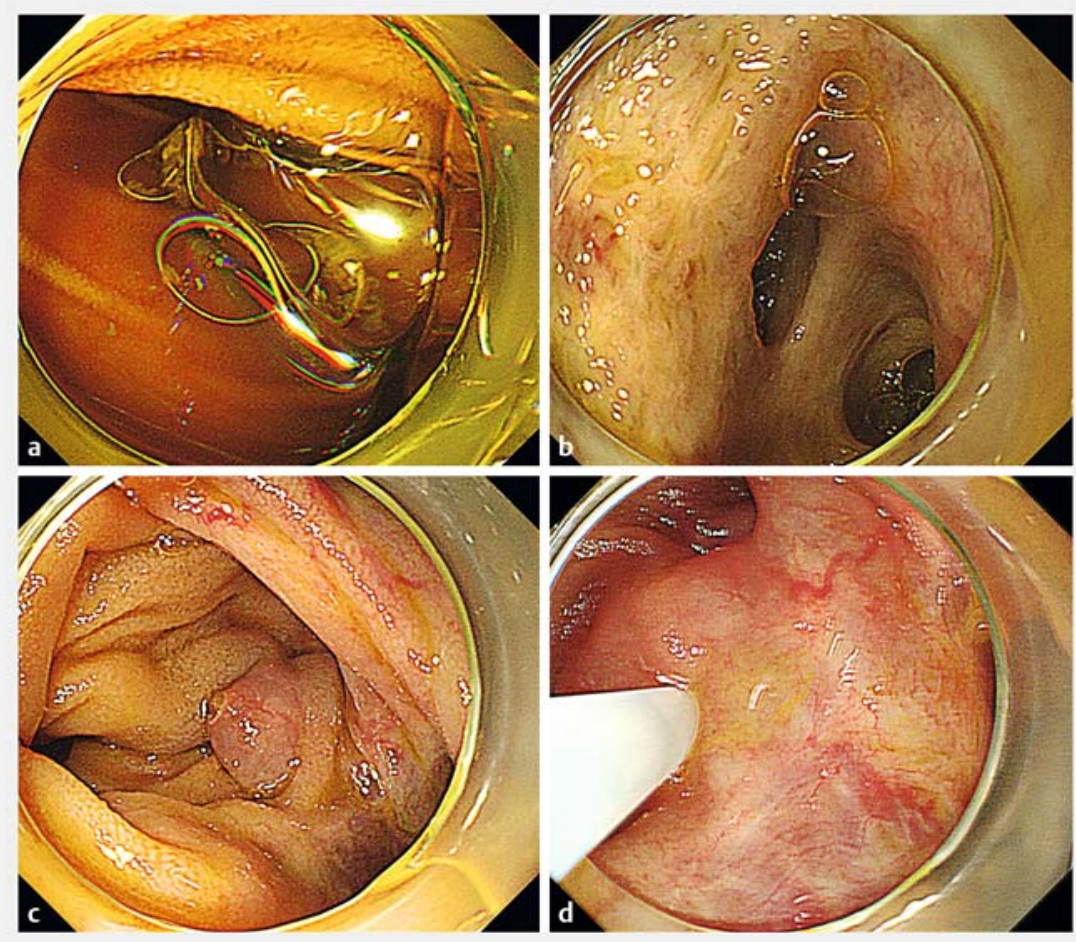

Fig. 2 Endoscopic images. a The coils, which were exposed near to the site of the choledocho-jejunostomy anastomosis. b Choledocho-jejunostomy anastomosis. c Jejunal varices with a fibrin plug (arrow). d Endoscopic injection sclerotherapy for jejunal varices.

\section{References}

[1] Sasamoto A, Kamiya J, Nimura Y et al. Successful embolization therapy for bleeding from jejunal varices after choledochojejunostomy: report of a case. Surg Today 2010; 40: $788-791$

[2] Saeki Y, Ide K, Kakizawa $\mathrm{H}$ et al. Controlling the bleeding of jejunal varices formed at the site of choledochojejunostomy: report of 2 cases and review of the literature. Surg Today 2013; 43: $550-555$

\section{Bibliography}

DOI https://doi.org/10.1055/a-0987-9780

Published online: 21.8.2019

Endoscopy 2020; 52: E41-E42

(c) Georg Thieme Verlag KG

Stuttgart · New York

ISSN 0013-726X

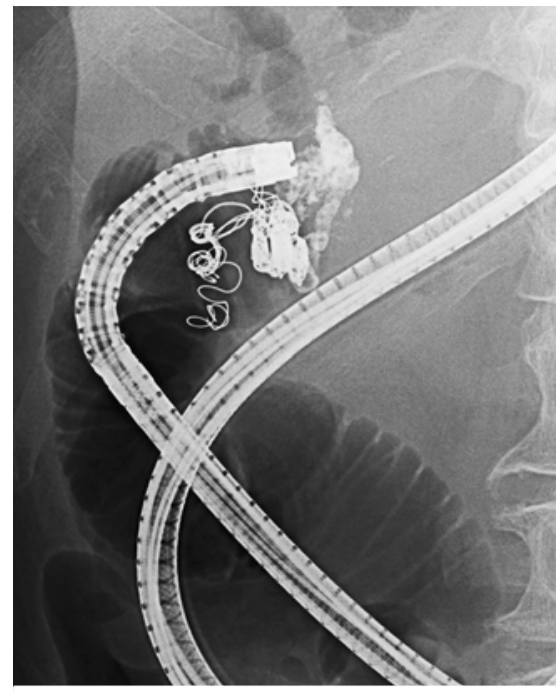

- Fig.3 After endoscopic injection sclerotherapy, the injectate was seen occupying the jejunal varices (arrow).

\section{ENDOSCOPY E-VIDEOS}

https://eref.thieme.de/e-videos

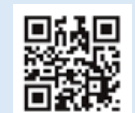

Endoscopy E-Videos is a free access online section, reporting on interesting cases and new

techniques in gastroenterological endoscopy. All papers include a high quality video and all contributions are freely accessible online.

This section has its own submission website at

https://mc.manuscriptcentral.com/e-videos 